\title{
The rectangular sector-by-technology model: not every economy produces every product and some products may rely on several technologies simultaneously
}

\author{
Faye Duchin • Stephen H Levine
}

Received: 5 March 2012 / Accepted: 17 April 2012 / Published online: 17 April 2012 (C) 2012 Duchin and Levine; licensee Springer. This is an Open Access article distributed under the terms of the Creative Commons Attribution License (http://creativecommons.org/licenses/by/2.0), which permits unrestricted use, distribution, and reproduction in any medium, provided the original work is properly cited.

\begin{abstract}
This paper identifies a fundamental challenge in the development of inputoutput databases of the world economy intended for analysis of alternative scenarios with a model of the world economy. Primary data sources for individual economies generally do not use the same sectoral classification schemes, in part for lack of coordination and also because not all economies produce all goods. In addition, some economies produce a given good simultaneously using several technologies, sometimes quite distinct in terms of factor requirements and cost structures, information that can and should be retained for scenario analysis. To accommodate the relevant information in a way that is both precise and parsimonious, we introduce rectangular input-output matrices and a modeling framework for analyzing them. This framework extends an existing input-output/linear programming model, the rectangular choiceof-technology (RCOT) model, and integrates it into an existing input-output/linear programming model of the world economy, the World Trade Model (WTM). The desirable properties of the resulting WTM/RCOT model are illustrated through a numerical example. This formulation requires that a criterion be specified to choose among available options, and we discuss some alternative criteria. Square inputoutput matrices and their inverses are only a special case of this more general formulation, and we show how moving to the rectangular formulation expands the types of questions that input-output analysts are able to address.
\end{abstract}

Keywords world input-output database $\cdot$ rectangular input-output matrices $\cdot$ choice of technology model $\cdot$ World Trade Model $\cdot$ linear programming model

F Duchin ( $\varangle)$

Department of Economics, Rensselaer Polytechnic Institute, Troy, NY 12180, USA

e-mail: duchin@rpi.edu

SH Levine

Department of Civil and Environmental Engineering, Tufts University, Medford, MA 02155, USA

e-mail: stephen.levine@tufts.edu 
JEL Classification C67 · C61 · F14 · O33

\section{Background}

With the launching of this journal, the Pan-Pacific Association of Input-Output Studies seeks to expand its influence to a global community of scholars and to extend its scope beyond what it calls (in the journal brochure) 'classical input-output economics' by encouraging new approaches to understanding and analyzing relationships among the components of contemporary economic systems. Global financial crisis, urgent environmental challenges, and widespread social unrest are compelling reasons not only to refine our analytic frameworks but also explicitly to hone them for addressing specific critical problems, to collaborate across disciplines for inventing solution concepts to deal with them, and to put our databases, methods, and models to work for the express purpose of evaluating realistic and potentially promising, actionable alternatives. Not surprisingly, there is now a resurgence of research on compiling input-output databases and creating input-output models of the world economy for undertaking the analysis of such scenarios.

Leontief et al. (1977) created the first input-output model of the world economy as well as the database to which it was applied. Today, there are freestanding inputoutput databases of the world economy that are available for use with a variety of models, including not only input-output models but also general equilibrium models. Several input-output databases that cover the entire world economy at substantial levels of regional and sectoral detail are in preparation; they include EXIOBASE (Tukker et al. 2009), Eora (Kanemoto et al. 2011), WIOD (WIOD 2011), and a widely-used one of earlier vintage, GTAP (Narayanan and Walmsley 2008). Among the input-output models, there is a convergence of regional and world modeling approaches and, especially, a sharing of common databases. Regional input-output analysis has, for decades, focused on multiplier analysis at the smaller than national level, the multipliers being derived from the inverse of a square matrix. By contrast, the input-output models of the world economy have been based on national spatial units and, while these models also used square matrices, it was for the purpose of deriving quantities of output and trade flows and changes in prices under alternative scenarios about the future rather than calculating multipliers. The emerging convergence is due in large measure to the common interest today in exchanges linking a variety of spatial units, such as river basins or urban areas and their hinterlands, in the context of both intra-country and multi-country regions.

This article proposes a solution to a particular problem that became apparent in the course of applying the World Trade Model (Duchin 2005), a linear programming input-output model of the world economy based on comparative advantage subject to resource constraints, to a new environmentally extended input-output database of the economy compiled from many sources at an unprecedented level of detail (EXIOBASE). While the problem is more general, it is readily explained by considering only the square (or 'symmetric') input-output tables representing the economies of the diverse countries and regions of the world. A typical requirement for databases of the world economy is that all tables have the same number $n$ of sectors and that the 
sectors be defined in comparable ways. Comparability is required to assure the consistency between quantities of goods produced in and exported from the economies of origin and the corresponding imports into the destination economies. The implicit underlying assumption is that every region produces all $n$ outputs, and each output is produced in a given region using a single, average input structure. Thus, if $n$ sectors are to be included in the database, every region will be represented by an input-output matrix, $\mathbf{A}$, with $n$ columns and $n$ rows. However, it is not evident what to put in those columns and rows if the original input-output table for this region does not identify the corresponding sectors, either because their products are not produced and possibly not even consumed in that region or because they were aggregated in that region's table with other sectors.

In the database in question, we encountered columns and rows of 0 in these cases. This treatment works as a passive placeholder from a descriptive point of view in that it maintains the formal structure of $n$ rows and $n$ columns, and it appears unproblematic in that it does not affect row totals or column totals in the input-output flow table. However, it poses a problem for scenario analysis as a column of coefficients that are all zeroes makes the region appear to have a comparative advantage (because of very low, in fact zero, input costs) in precisely those sectors where it obviously does not. As a temporary fix, we replaced the column of coefficients by equally artificial, large entries (in contrast to the artificially low ones) to be sure the model did not interpret the region as a low-cost producer. This paper proposes a much more precise and parsimonious representation than the trick of fictive columns with extreme values and one that turns out to offer other advantages as well.

We make two assertions about a database with more than a handful of sectors, and contemporary world input-output databases aim at many dozens if not hundreds of sectors. First, at least some of the regions will not be equipped to produce some products, and second, for some sectors and regions, it is important to distinguish alternative technologies that both compete with each other for limited resources and may be simultaneously in use. Examples of the former are the extraction of petroleum or rare earth metals, which are obviously concentrated in certain regions and cannot, for physical reasons, be extracted in others even though the resource commodity may be imported and used there. Examples of multiple, potentially simultaneously utilized technological options that need to be distinguished include grain production on rainfed and on irrigated land or the generation of electric power using coal and nuclear fuels, since the options differ in virtually all attributes (namely cost structure, resource requirements, and environmental impact) except that they produce indistinguishable outputs. Naturally, all sectors whose outputs are tradable require that the sectoral output be defined similarly (in terms of a representative output or product mix) in all regions. In what follows, we assume a world economy with $n$ sectors producing potentially tradable goods and services in some, but not necessarily all regions.

In an earlier paper, we introduced the rectangular choice-of-technology (RCOT) model (Duchin and Levine 2011) for allowing several choices of input-cost structures in a given sector and demonstrated its use both in a model of a single region and in the context of a world economy consisting of several regions. RCOT is a linear program with both a primal quantity model that solves for output, trade flows, and factor use, and a dual model that determines product prices and scarcity rents on fully utilized 
factors of production. This RCOT model assumed the same $n$ sectors in all regions but allowed any number of alternative input structures for each sector in a given region. The RCOT model replaces the familiar square matrices A (the matrix of intermediate coefficients per unit of output) and $\mathbf{I}$ (the identity matrix) by rectangular matrices, $\mathbf{A}^{*}$ and $\mathbf{I}^{*}$. In this paper, we generalize these rectangular matrices to accommodate the important case where a given region has zero options - rather than one or more than one - for producing the product of a particular sector, that is, it is not equipped to produce that output at all. In this case, there is no information for creating a column for this sector, and we will see that a column is not needed. The region may, however, require the use of the output in question, which then it must import; if it does not use the product either, the demand quantified in the row of intermediate inputs is legitimately and unproblematically zero. That is, whether or not the product is used in the region, the row for this sector need not be suppressed. The rectangularity of the regional matrices comes from the fact that each one has exactly $n$ rows but may have a smaller or larger number of columns. The number of columns is reduced by each sector that does not produce in that region and increased for each sector that has more than one technology option. Note that even if the rectangular matrix happens to be square (i.e., with the same number of rows and columns), this does not assure a one-to-one relationship between rows and columns as in the standard A matrix. It may mean, for example, that one sector is not present in this economy, but another sector has two technological options.

In this paper, we extend the RCOT representation to include the absence of technological options for a sector (i.e., no capacity to produce), a true generalization of the original case (Duchin and Levine 2011) of 1 through $s$ technological options for a sector to the case of 0 through $s$ options. We also fully incorporate the extended RCOT model into the World Trade Model. The extended World Trade Model (WTM/RCOT) representation has the new feature that a region may use identifiable imported inputs that it cannot produce itself, the so-called non-competitive imports, that typically are vital for an economy such as resource commodities or capital goods. While non-competitive imports are often aggregated by money value into a single category in one-region input-output tables, a WTM/RCOT analysis permits them to be explicitly identified. An economy with many non-competitive imports will generally be represented by a rectangular input-output matrix with more rows than columns.

The rest of this paper is divided into four sections. In the following sections we describe the rectangular input-output matrices, $\mathbf{A}^{*}$ and $\mathbf{I}^{*}$. Next, the algebra for the rectangular World Trade Model, with rectangular sector-by-technology matrices for all regions, is developed for the primal quantity model and illustrated by a numerical example for three regions with three sectors and four factors of production. The model and numerical solutions for the dual price model are also presented. Section 3 concludes.

\section{Results and discussion}

\subsection{The rectangular matrices $\mathbf{A}^{*}$ and $\mathbf{I}^{*}$}

The rectangular $\mathbf{A}^{*}$ matrix for a region's economy is readily visualized. It contains $n$ rows, and for each row, there are 0 or more columns of coefficients representing 
alternative ways of producing the output of the row sector. In the basic input-output equation, $(\mathbf{I}-\mathbf{A}) \mathbf{x}=\mathbf{y}$, the identity matrix I seems to play a trivial role. However, the placement of $1 \mathrm{~s}$ at the intersection of the $i$ th row with the $i$ th column, that is, down the diagonal - and 0 elsewhere - in fact provides the vital function of assuring that the output produced by the $i$ th column technology in $\mathbf{A}$ is delivered to the using sectors in the amounts specified by the entries in the $i$ th row of $\mathbf{A}$. The generalized matrix $\mathbf{I}^{*}$ exhibits a similar but more complex logic. It, too, contains $n$ rows, one for each sector. However now, a given row contains a 1 for each column technology that may be used to produce the output of this sector and 0 elsewhere. While I contains one and only one instance of 1 in each row, a row of $\mathbf{I}^{*}$ may contain 0,1 , or any number of $1 \mathrm{~s}$, with a row of all 0 indicating that the corresponding sector is absent from that region. Thus, $\mathbf{I}$ is a special case of $\mathbf{I}^{*}$. As already noted, when $\mathbf{I}^{*}$ is square but not equal to $\mathbf{I}$, then there must be at least one row with only 0 s and at least one other with two or more $1 \mathrm{~s}$.

The database intended for scenario analysis requires several matrices and vectors in addition to $\mathbf{I}^{*}$ and $\mathbf{A}^{*}$. Besides the vector, $\mathbf{y}$, of domestic demand, we introduce $\mathbf{F}^{*}$, the matrix of factor input requirements per unit of output; the vector of factor endowments, $\mathbf{f}$; and the vector of unit prices of factors, $\boldsymbol{\pi}$. The quantity model is solved for $\mathbf{x}^{*}$, output by technology, and $\mathbf{x}$, output by sector.

We assume a world economy of $m$ regions in which $n$ goods and services are produced. The total number of distinct factors of production that are utilized in all regions combined is $k$, of which $k_{r}$, where $k_{r} \leq k$, are available in region $r$. Since factors are not traded, they need not be defined in the same ways in different regions. Thus, each region may produce up to $n$ goods using up to $k$ factors of production, using alternative technologies for producing any given output. We denote by $s_{r i}$ the number of alternatives in region $r$ for producing the output of sector $i$, and $s_{r}=$ $\sum_{i} s_{r i}$ is the total number of technologies available in region $r$. The matrices and variables have the following dimensions:

$$
\begin{array}{ll}
\mathbf{I}_{r}^{*}, \mathbf{A}_{r}^{*} & n \times s_{r} \\
\mathbf{F}_{r}^{*} & k_{r} \times s_{r} \\
\mathbf{f}_{r}, \boldsymbol{\pi}_{r} & k_{r} \times 1 \\
\mathbf{y}_{r}, \mathbf{x}_{r} & n \times 1 \\
\mathbf{x}_{r}^{*} & s_{r} \times 1 .
\end{array}
$$

Note that each rowsum of $\mathbf{I}_{r}^{*}$ is equal to $s_{r i}$, the total number of technological options for sector $i$ in region $r$. All the matrices with the superscript * have as many columns as the corresponding region, $r$, has technologies. The matrices $\mathbf{I}^{*}$ and $\mathbf{A}^{*}$ have as many rows, $n$, as there are distinct sectors globally (i.e., the union of the set of sectors in each region) and as many columns as there are technological options in the region. Global sectors that are absent in a region are indicated by rows of 0 in the region's $\mathbf{I}^{*}$ matrix. The matrix $\mathbf{F}^{*}$ has as many rows, $k_{r}$, as there are factors in the region and as many columns as $\mathbf{I}^{*}$ and $\mathbf{A}^{*}$. Similarly, all the vectors with superscript ${ }^{*}$ have as many elements as the corresponding region has technologies. Note that all vectors and matrices are in boldface. When indexed, whether by region or other attribute, those indices are part of the vector or matrix name and therefore are in boldface as well. The elements of these matrices, being scalars, are not in boldface. Therefore, 
the index $\mathbf{i}$ in the matrix $\mathbf{A}_{\mathbf{i}}$ is in boldface while the element in its $m$ th row and $n$th column is $a_{i j, m n}$.

A numerical example of the original RCOT model showing the case of a region with one or more options for producing each good, both in the one-region case and in the context of a three-region world economy, is provided in Duchin and Levine (2011). In the following section, we extend the generality of the World Trade Model by incorporating an extended version of RCOT and provide a numerical example for a global system with trade based on regional comparative advantages, where a region may have zero technological options for producing the output of one or more sectors.

\subsection{Extending the rectangular World Trade Model}

The World Trade Model is a linear program with a quantity primal and a price dual (Duchin 2005). In this section, we show the primal; it determines the inter-regional division of labor that assures that exogenous domestic final demand is satisfied in all regions according to a decision criterion represented by the objective function. We choose a criterion to minimize global factor usage, where the amount of factor usage in each region is weighted by region-specific factor prices. This decision contrasts with the common practice in economic optimization models of maximizing consumption (or 'utility'). The WTM is written below in terms of $\mathbf{I}^{*}, \mathbf{A}^{*}, \mathbf{F}^{*}$, and $\mathbf{x}^{*}$ in place of $\mathbf{I}, \mathbf{A}, \mathbf{F}$, and $\mathbf{x}$, permitting an economy to have zero options for some sectors and two or more for others:

$$
\begin{aligned}
& \min Z=\sum_{r} \boldsymbol{\pi}_{r}^{T} \mathbf{F}_{r}^{*} \mathbf{x}_{r}^{*} \\
& \text { s.t. } \quad \sum_{r}\left(\mathbf{I}_{r}^{*}-\mathbf{A}_{r}^{*}\right) \mathbf{x}_{r}^{*}=\sum_{r} \mathbf{y}_{r} \\
& \mathbf{F}_{r}^{*} \mathbf{x}_{r}^{*} \leq \mathbf{f}_{r} \quad \forall r \\
& \mathbf{x}_{r}^{*} \geq \mathbf{0} \quad \forall r .
\end{aligned}
$$

Below is a numerical example that illustrates the properties of the extended WTM/RCOT model for a three-region $(r=3)$ world economy with a total of three sectors $(n=3)$ and four factors $(k=4)$ of production. The regions are as follows:

1. Industrialized

2. Agricultural

3. Non-industrialized with mineral resources.

The sectors producing goods and services are as follows:

1. Agriculture

2. Manufacturing

3. Mining.

The factors of production are as follows:

1. Labor

2. Capital

3. Ore

4. Land. 
For simplicity, we will assume the same factors in all economies. Numerical values for the variables and parameters are given as follows:

- Region 1: industrialized

$$
\begin{aligned}
& s_{1}=3 \\
& s_{11}=1, \quad s_{12}=2, \quad s_{13}=0 \\
& k_{1}=3 \\
& \mathbf{I}_{1}^{*}=\left[\begin{array}{lll}
1 & 0 & 0 \\
0 & 1 & 1 \\
0 & 0 & 0
\end{array}\right], \quad \mathbf{A}_{1}^{*}=\left[\begin{array}{ccc}
0.13 & 0.08 & 0.01 \\
0.25 & 0.5 & 0.6 \\
0.4 & 0.3 & 0.3
\end{array}\right], \\
& \mathbf{F}_{1}^{*}=\left[\begin{array}{ccc}
1.75 & 0.8 & 0.1 \\
3 & 1 & 2 \\
2 & 0 & 0
\end{array}\right] \\
& \mathbf{f}_{1}=\left[\begin{array}{c}
150 \\
325 \\
70
\end{array}\right], \quad \mathbf{y}_{1}=\left[\begin{array}{l}
10 \\
20 \\
10
\end{array}\right], \quad \pi_{1}=\left[\begin{array}{l}
3 \\
2 \\
5
\end{array}\right] .
\end{aligned}
$$

- Region 2: agricultural

$$
\begin{aligned}
& s_{2}=2 \\
& s_{21}=1, \quad s_{22}=1, \quad s_{23}=0 \\
& k_{2}=4 \\
& \mathbf{I}_{2}^{*}=\left[\begin{array}{ll}
1 & 0 \\
0 & 1 \\
0 & 0
\end{array}\right], \quad \mathbf{A}_{2}^{*}=\left[\begin{array}{ll}
0.2 & 0.1 \\
0.1 & 0.5 \\
0.2 & 0.3
\end{array}\right], \quad \mathbf{F}_{2}^{*}=\left[\begin{array}{cc}
8 & 30 \\
4 & 0 \\
0 & 0 \\
10 & 2
\end{array}\right] \\
& \mathbf{f}_{2}=\left[\begin{array}{l}
800 \\
300 \\
100 \\
600
\end{array}\right], \quad \mathbf{y}_{2}=\left[\begin{array}{c}
30 \\
10 \\
5
\end{array}\right], \quad \boldsymbol{\pi}_{2}=\left[\begin{array}{c}
0.5 \\
2 \\
5 \\
0.4
\end{array}\right] .
\end{aligned}
$$

- Region 3: mineral rich

$$
\begin{aligned}
& s_{3}=2 \\
& s_{31}=1, \quad s_{32}=0, \quad s_{33}=1 \\
& k_{3}=4 \\
& \mathbf{I}_{3}^{*}=\left[\begin{array}{ll}
1 & 0 \\
0 & 0 \\
0 & 1
\end{array}\right], \quad \mathbf{A}_{3}^{*}=\left[\begin{array}{cc}
0.3 & 0 \\
0.4 & 0.3 \\
0.5 & 0.05
\end{array}\right], \quad \mathbf{F}_{3}^{*}=\left[\begin{array}{cc}
10 & 0.25 \\
1 & 1 \\
0 & 1.5 \\
5 & 0
\end{array}\right] \\
& \mathbf{f}_{3}=\left[\begin{array}{c}
30 \\
100 \\
150 \\
200
\end{array}\right], \quad \mathbf{y}_{3}=\left[\begin{array}{c}
8 \\
10 \\
6
\end{array}\right], \quad \boldsymbol{\pi}_{3}=\left[\begin{array}{l}
1 \\
4 \\
2 \\
2
\end{array}\right] .
\end{aligned}
$$


The I* matrix for each region reveals three properties: the number of rows corresponds to the number of intermediate inputs, the presence of 1 in a row indicates that a particular input can be produced domestically, and more than one occurrence of 1 in the row means that there is more than one technology for producing it domestically. In the numerical example, the industrialized region requires the output of the mining sector as an intermediate input; however, it has no mining sector as it has no ore reserves. This region has an agricultural sector and two different manufacturing technologies. The second region is endowed with land and labor and has the ability to produce both agricultural and manufactured products. It possesses ore reserves but has no mining industry. The final region is endowed with ore and has both mining and agricultural sectors but does not have manufacturing capacity in place.

The results of the WTM computation show the following pattern of specialization in terms of four vectors, where $\mathbf{x}^{*}$ is the vector of output by technology and $\mathbf{x}$ is the output by sector. From these results, we can also calculate two additional vectors: $\mathbf{e}$ is the $n \times 1$ vector of net exports (calculated as the difference between production and the sum of intermediate and final demand) and $\varphi$ is the $k_{r} \times 1$ vector of factor use (calculated as $\mathbf{F}^{*} \mathbf{x}^{*}$ ):

$$
\begin{aligned}
& \mathbf{x}_{1}^{*}=\left[\begin{array}{c}
5.72 \\
45.88 \\
130.97
\end{array}\right], \quad \mathbf{x}_{1}=\left[\begin{array}{c}
5.72 \\
176.85 \\
0
\end{array}\right], \quad \mathbf{e}_{1}=\left[\begin{array}{c}
-10 \\
53.9 \\
-65.35
\end{array}\right], \quad \boldsymbol{\varphi}_{1}=\left[\begin{array}{c}
59.82 \\
325 \\
11.45
\end{array}\right] \\
& \mathbf{x}_{2}^{*}=\left[\begin{array}{c}
60 \\
0
\end{array}\right] \\
& \mathbf{x}_{2}=\left[\begin{array}{c}
60 \\
0 \\
0
\end{array}\right] \\
& \mathbf{e}_{2}=\left[\begin{array}{c}
18 \\
-16 \\
-17
\end{array}\right] \\
& \boldsymbol{\varphi}_{2}=\left[\begin{array}{c}
480 \\
240 \\
0 \\
600
\end{array}\right] \\
& \mathbf{x}_{3}^{*}=\left[\begin{array}{c}
0 \\
93.0
\end{array}\right] \\
& \mathbf{x}_{3}=\left[\begin{array}{c}
0 \\
0 \\
93.0
\end{array}\right], \\
& \mathbf{e}_{3}=\left[\begin{array}{c}
-8 \\
-37.9 \\
82.34
\end{array}\right] \text {, } \\
& \boldsymbol{\varphi}_{3}=\left[\begin{array}{c}
23.25 \\
93.0 \\
139.49 \\
0
\end{array}\right] \text {. }
\end{aligned}
$$

The division of labor among the three regions that corresponds to the lowest global factor costs has the third region specializing in mining and the second region in agricultural production. The first region specializes in manufacturing but is also obliged to produce some agricultural output to supplement its imports from region 2 . The reason is that region 2 , the relatively lowest-cost producer, has run into a land constraint (the 600 units of land available according to $\mathbf{f}_{2}$ being fully utilized as shown by the last component of $\varphi_{2}$ that is also 600) and is therefore not able to satisfy total world demand for food.

While region 1 is the only producer of manufactured goods, it needs to deploy both of its manufacturing technologies for this purpose. The capital-intensive technology (with the higher capital to labor requirements according to the first two rows of $\mathbf{F}^{*}$ ) is the less costly option, but the region runs into a capital constraint (seen by comparing the second components of $\mathbf{f}_{1}$ and $\boldsymbol{\varphi}_{1}$ ) and needs in addition to operate its labor-intensive manufacturing technology to satisfy the production requirements.

This example demonstrates the operational nature of the combined RCOT World Trade Model, allowing a determinate solution when regions have different numbers of sectors and factor endowments. It also shows the double functioning of the factor 
constraints in physical units, both to require more than one region to produce a given good and to require a region to simultaneously utilize more than one technology, in sectors where the relatively lowest-cost alternative runs into a factor constraint.

\subsection{The price dual}

The dual price model determines the world prices for traded goods and scarcity rents of factors that are fully utilized. It is a distinctive feature of the WTM that factors have two-part prices: an exogenously given nominal price, received by the owner whether or not the factor is fully utilized, and an endogenous scarcity rent that is non-zero if it is fully utilized and zero otherwise (see Duchin and Levine 2011 for a more extended discussion of the two-part prices). The dual results take the form of two additional endogenous variables: $\mathbf{p}_{\mathbf{0}}$, the $n \times 1$ vector of world prices, and $\rho_{\mathbf{r}}$, the $k_{r} \times 1$ vectors of region-specific scarcity rents. The price model is written as follows:

$$
\begin{aligned}
& \max \mathrm{W}=\sum_{r}\left(\mathbf{y}_{r}^{T} \mathbf{p}_{\mathbf{0}}-\mathbf{f}_{r}^{T} \boldsymbol{\rho}_{\mathbf{r}}\right) \\
& \text { s.t. } \quad\left(\mathbf{I}_{r}^{*}-\mathbf{A}_{r}^{*}\right)^{T} \mathbf{p}_{\mathbf{0}}-\mathbf{F}_{r}^{* T} \rho_{\mathbf{r}} \leq \mathbf{F}_{r}^{* T} \boldsymbol{\pi}_{r} \quad \forall r \\
& \mathbf{p}_{\mathbf{0}} \geq \mathbf{0} \\
& \boldsymbol{\rho}_{\mathbf{r}} \geq \mathbf{0} \quad \forall r .
\end{aligned}
$$

The price inequality for a particular sector is binding for regions that actually produce, but it has a non-zero slack for other regions (see Duchin 2005 for the proof). Given the form of the inequality, the world price for a good is set at the cost of the highest-cost producer that actually enters into production. Thus, the lower-cost producers, whose output was necessarily limited by a factor constraint, earn a rent on that scarce factor that is equal to the difference between the world price and their own (lower) cost of production. The scarcity rent measures by how much total factor costs (i.e., the value of the objective function of the primal) could be reduced if one more unit of the scarce factor was available. It is well known that at the optimal solution, the primal and dual objective functions have the same value, $Z=W$, assuring that the payments for final deliveries equal the total earnings of the factors of production.

The solution to the price dual for the numerical example is as follows:

$$
\mathbf{p}_{\mathbf{0}}=\left[\begin{array}{l}
40.21 \\
25.38 \\
15.64
\end{array}\right]
$$

and

$$
\rho_{\mathbf{1}}=\left[\begin{array}{c}
0 \\
0.38 \\
0 \\
0
\end{array}\right], \quad \rho_{\mathbf{2}}=\left[\begin{array}{c}
0 \\
0 \\
0 \\
1.05
\end{array}\right], \quad \boldsymbol{\rho}_{\mathbf{3}}=\left[\begin{array}{l}
0 \\
0 \\
0 \\
0
\end{array}\right] .
$$

The existence of two non-zero scarcity rents reflects the already noted facts that capital is scarce in region 1; land, in region 2 . Note that an additional unit of land in region 2 , allowing it to produce more agricultural output in place of region 1 , would reduce global factor costs by more than an additional unit of capital in region 1 (1.05 vs. 0.38 money units), allowing the latter to increase production with its more capitalintensive technology in place of the more labor-intensive option. 


\section{Conclusions}

The original rectangular choice-of-technology model with one or more technological options for each sector is readily implemented for a single region, and it introduces additional flexibility into a one-region analysis that permits addressing a wider set of questions. However, the reader will note that the prospect of zero technological options was illustrated only for the case of the world model. While it would be useful in the one-region case also to explicitly itemize the non-competitive imports, doing so would require an additional extension to the model that we do not undertake here. The defining characteristic of a one-region model is that trade flows must be provided exogenously. However, an exogenous value for imports of the non-competitive goods would not, in general, be consistent with endogenously determined demand for these goods under different scenarios. Thus, an additional mechanism for endogenous determination of imports under alternative scenarios would be needed. A fully elaborated way of providing that function in the general case is precisely the input-output model of the world economy.

The compilation of input-output databases for the world economy is now sufficiently mature that definitions and conventions are needed to assure compatibility of the data taken from various sources, at a minimum among the input-output tables prepared by individual national statistical offices. Requiring that all use the same classifications for intermediate and factor inputs is overly constraining, however, because in reality they do not all produce and use the same goods and services, and there is no reason to assume that they will in the future. Instead, as we have shown, it is possible to introduce flexibility in two ways. First, it is adequate to require common definitions for tradable goods and services only, and a precise and parsimonious representation is achieved by simply omitting columns for sectors that are, in fact, non-existent. Second, the existence of simultaneously utilized technologies in place for producing a given good with sharply distinct input structures (e.g., nuclear fuel vs. coal or wind power for generating electricity) can and should be reflected. In fact, such information is often included in the rectangular USE matrices compiled in many national statistical offices and should be retained. The fundamental contribution of the RCOT model is to demonstrate that the general form of an input-output system is based on rectangular matrices, a sharp departure from the standard, and unnecessarily limiting, conviction that an input-output model requires a square, invertible matrix.

Databases of the world economy are utilized with models of the world economy, and the models need to be able to manipulate these rectangular matrices. We have demonstrated the algebra for easily accommodating the absence of particular sectors in a region and the choice among alternative technologies through the replacement of the identity matrix $\mathbf{I}$ by the more general $\mathbf{I}^{*}$ matrix and the square $\mathbf{A}$ matrix by $\mathbf{A}^{*}$. To choose among alternative technologies is a task for the model, not the database. Input-output models have not, in the past, required establishing a criterion for choice. One possible choice, of course, is to maximize consumption for given availability of factors. This approach promotes full utilization of all factors of production, not only labor and built capital but also reserves of resources, land, and freshwater. Another, which we favor, is to minimize factor use to satisfy given consumption requirements and, under alternative scenarios, to examine how pollution and pressure on resources 
might respond to changes not only in technologies and endowments but also in consumption in a framework that does not assume that more consumption or more factor use is necessarily better. Imposing a criterion for choice thus extends the reach of input-output models for analyzing alternative approaches to addressing the most pressing contemporary challenges regarding sustainable development.

\section{Competing interests}

The authors declare that they have no competing interests.

\section{References}

Duchin F (2005) A World Trade Model based on comparative advantage with $\mathrm{m}$ regions, $n$ goods, and $k$ factors. Econ Syst Res 17(2):1-22

Duchin F, Levine SH (2011) Sectors may use multiple technologies simultaneously: the rectangular choiceof-technology model with binding factor constraints. Econ Syst Res 23(3):281-302

Kanemoto K, Lenzen M, Geschke A, Moran D (2011) Building Eora: a global multi-region input-output model at high country and sector [disaggregation]. Presented at the 19th international input-output conference, Alexandria, USA, 13-17 June 2011

Leontief W, Carter AP, Petri PA (1977) The future of the world economy. Oxford University Press, New York

Narayanan BG, Walmsley TL (2008) Global trade, assistance, and production: the GTAP 7 data base. Center for Global Trade Analysis, Purdue University. https://www.gtap.agecon.purdue.edu/databases/v7/ v7_doco.asp. Accessed 11 March 2012

Tukker A, Poliakov E, Heijungs R, Hawkins T, Neuwahl F, Rueda-Cantouche J, Giljum S, Moll S, Oosterhaven J, Bouwmeester M (2009) Towards a global multiregional environmentally-extended inputoutput database. Ecol Econ 68(7):1928-1937

WIOD (2011) The WIOD-Database. http://www.wiod.org/database/index.htm. Accessed 11 March 2012 